\title{
A 2D/3D-QSAR STUDY ON BIOLOGICAL ACTIVITIES OF 1,2-ETHYLENDIAMINE DERIVATIVES AS ANTI-TUBERCULOSIS DRUGS
}

\author{
GHASEM GHASEMI*, REIHANEH MOHAMADZADE \\ Department of Chemistry, Rasht Branch, Islamic Azad University, Rasht, Iran
}

\begin{abstract}
In this work quantitative structure-activity relationship (QSAR) study has been done on 1,2-ethylenediamine derivatives as anti-tuberculosis drugs. Genetic algorithm (GA), artificial neural network (ANN), multiple linear regressions (stepwise-MLR) and Imperialist Competitive Algorithm (ICA), were used to create the nonlinear and linear QSAR models. The root-mean square errors of the training set and the test set for GA-ANN models using the jack-knife method, were $0.1402,0.1304$ and $\mathrm{Q}^{2}=0.94$. Also, the $\mathrm{R}$ and $\mathrm{R}^{2}$ values $0.85,0.73$ in the gas phase were obtained from a GA-stepwise-MLR model. Q2 of training set for PLS was 0.52. The results obtained from this work indicate that ANN and ICA models are more effective than other statistical methods and exhibit reasonable prediction capabilities. The best descriptors are G3u, HATS2e, F02(C-N), GGI10, RDF040m, Mor22p, Mor05p, TIC4, H4e, H-052, G2m and G1e.
\end{abstract}

KEYWORDS: Tuberculosis, quantitative structure-activity relationship, 1,2-ethylenediamine derivatives, Genetic Algorithm and Imperialist Competitive Algorithm, Artificial Neural Network

\section{INTRODUCTION}

Tuberculosis in humans is mainly caused by Mycobacterium tuberculosis ${ }^{1}$. The infection is transmitted by respirable droplets generated during forceful expiratory activity such as coughing.

Tuberculosis infection can be either active or latent. The World Health Organization (WHO) estimates that within the next 20 years about 30 million people will be infected with the bacillus. The clinical management of TB has relied heavily on a limited number of drugs such asisonicotinic acid, hydrazide, rifampicin, ethambutal, streptomycin, ethionamide, pyrazinamide, fluroquinolones $\operatorname{etc}^{3-4}$

In imperialistic competition algorithm(ICA), all the empires try to take possession of colonies of other empires and control them. This competition gradually brings about a decrease in the power of weaker empires and an increase in the power of more powerful ones. This competition is modelled by just picking some (usually one) of the weakest colonies of the weakest empires and making a competition among all empires to possess the colony, or colonies $5-7$.

The search for quantitative relations between chemical structure and biological activity is the subject of quantitative structure-activity relationships, the purpose of which is to explain why a given drug produces its particular effect, and ultimately to predict the effect of newly synthesized chemical compounds.

One of the important features of mathematical model is its ability to predict the activity of molecules not yet synthesized or those with limited in vivo and in vitro experimental information, for economic or ethical reasons.

Partial least squares (PLS), is highly sensitive to extreme values of variables, which do not contribute to a predictive model ${ }^{8}$. The situation becomes worse when more variables are introduced to the models. Thus, the larger the number of variables, the less the predicted value of the developed mode $1^{9}$. Therefore, a variable selection step is necessary prior to building PLS models. To solve this problem, GA and PLS have been combined in a variable selection of QSAR and QSPR modelling. Genetic algorithms are best known for their ability to efficiently search large spaces and have been widely applied in different fields ${ }^{10-11}$. Thus, GA can be used for improving the prediction of QSAR modelling.

The more commonly used techniques in construction of QSAR models are PLS, principle component regression (PCR) and ANN ${ }^{12-14}$. PLS is insensitive to co-linearity among the predictor variables and allows one to handle data sets where the number of variables is larger than the number of observations. Thus, for large data sets PLS is preferable. In addition, PLS analysis provides equations describing the relationship between one or more dependent variables and a group of explanatory variables.

The RMSE can be calculated for prediction or validation samples (RMSEP) and for calibration samples (RMSEC). RMSEC (all validation methods) is calculated as:

$$
\text { RMSEC }=\frac{1}{\text { yWeight }} \sqrt{\text { ResYCalVar }}
$$

Res Ycal Var is Residual calibration variance

\section{DATA SET}

The data set consists of the experimental bioavailabilities of 21 structurally-diverse chemicals as reported by Protopopova ${ }^{15}$. Twenty-seven compounds with MICs of $15.6 \mathrm{mM}$ were tested on Vero cells to determine in vitro cytotoxicity $\left(\mathrm{IC}_{50}\right)$ and to establish a selectivity index (SI). Five of the most potent compounds were tested for in vivo efficacy in a murine model of chronic tuberculosis infection.

\section{MATERIALS AND METHOD}

Actual half-maximal inhibitory concentration $\left(\mathrm{IC}_{50}\right)$ values of all compounds were selected from literature. This set contained the effective concentration activities of 21 diketo analogues. A set of seven compounds was randomly removed from the dataset to be used as the prediction set (PSET). The $\log \left(1 / \mathrm{IC}_{50}\right)$ of this set spanned the entire dataset. The remaining compounds were utilized as the training set (TSET). In the simplest form of bootstrapping, instead of repeatedly analyzing subsets of the data, sub samples of the data are repeatedly analyzed. Each sub sample is a random sample with replacement from the full sample.

The structure and biological data of 21 molecules were obtained from literature ${ }^{15}$. The 3D structures of the molecules were generated using the built optimum option of Chemoffice. The structures were then fully optimized based on the ab initio method using the DFT level of theory (Figure 1). Dragon (version 5.5) was employed to calculate the molecular descriptors. All calculations were performed using the Gaussian $09 \mathrm{~W}$ program series. Geometric optimization of compounds was carried out using the B3LYP method employing a 6-31G (2d) basis set.

The independent variables were molecular descriptors and the dependent variables were the actual half-maximal inhibitory concentration $\left(\mathrm{IC}_{50}\right)$ values. More than 3226 theoretical descriptors were selected and calculated. Unscrambler (version 9.7) was used for analysis of data and statistical methods. For each compound in the training sets, a correlation equation was derived using the same descriptors. The equation was then used to predict $\log \left(1 / \mathrm{IC}_{50}\right)$ values for the compounds from the corresponding test sets.

Stepwise MLR, GA, ANN and ICA were used to select the most appropriate descriptor from all descriptors.

A genetic algorithm (Figure 2, Table3), ICA (Figure 2,3), ANN, MLR (Table 2), PLS (Table 1), Principal component regression(Table1) and least absolute shrinkage and selection operator (Table 2) were used to create the QSAR models.

First, descriptors that had the same values for at least $75 \%$ of compounds within correlation coefficients less than 0.4 with the dependent variable were regarded redundant and removed. Finally, since highly correlated descriptors provide approximately identical information, a pairwise correlation was performed. When their correlations coefficient exceeded 0.95 , one of two descriptors was randomly removed. These descriptors would be used as inputs of the ANN. GA was utilized as the mean for non-linear feature selection. 
1<smiles>CC(C)CCCCNCCNC1C2CC3CC(C2)CC1C3</smiles><smiles>c1ccc(C(CNCCNCC23CC4CC(CC(C4)C2)C3)c2ccccc2)cc1</smiles>

4<smiles>[Y]C(NCC(c1ccccc1)c1ccccc1)NCC1CCC2CC1C2(C)C</smiles>

7<smiles>CC1C2CCC(CNCCNC3CCC4(C)C(C)CCC34)C1C2(C)C</smiles>

10

13<smiles>C1CCCC(NCCNC2C3CC4CC(C3)CC2C4)CC1</smiles>

16<smiles>COc1ccccc1CCNCCNC1C2CC3CC(C2)C1C3</smiles>

19<smiles>c1ccc(C(CCNCCNCC23CC4CC(CC(C4)C2)C3)c2ccccc2)cc1</smiles>

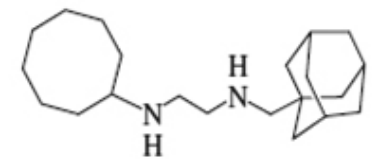

5<smiles>CC1C(NCCNCC(c2ccccc2)c2ccccc2)C2CCC1C2(C)C</smiles>

8<smiles>c1ccc(C(CNCCNC2CCCCCC2)c2ccccc2)cc1</smiles>

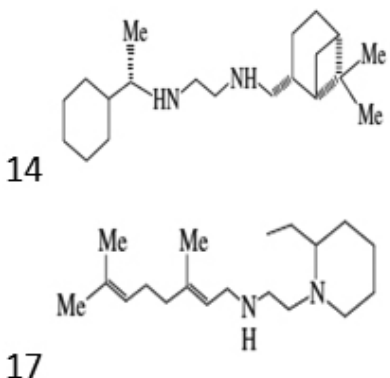

17

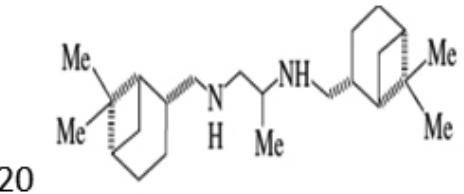<smiles>CC1C2CCC(C)(C1NCCNCCC(c1ccccc1)c1ccccc1)C2(C)C</smiles>

6

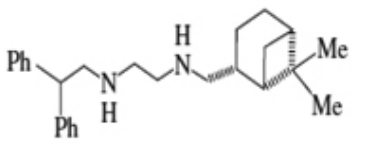<smiles>CC1CCCC(NCCNCC2CCC3CC2C3(C)C)C1C</smiles>

9

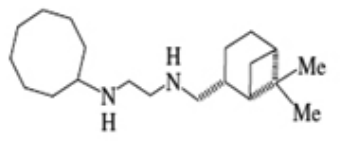

12

15

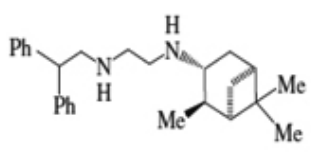

18

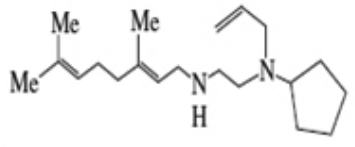

Fig. 1 The molecular structure of ethylenediamine analogues.

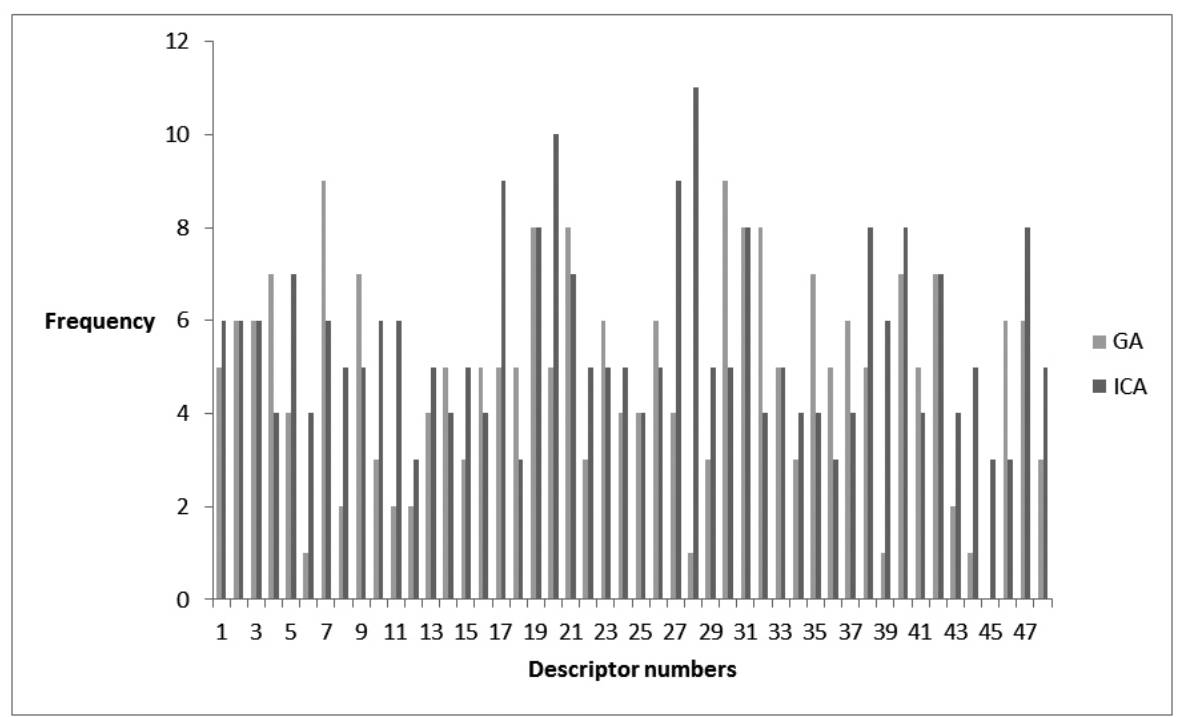

Fig. 2. The results of GA and ICA 
Training: $\mathbf{R}=\mathbf{0} .73787$

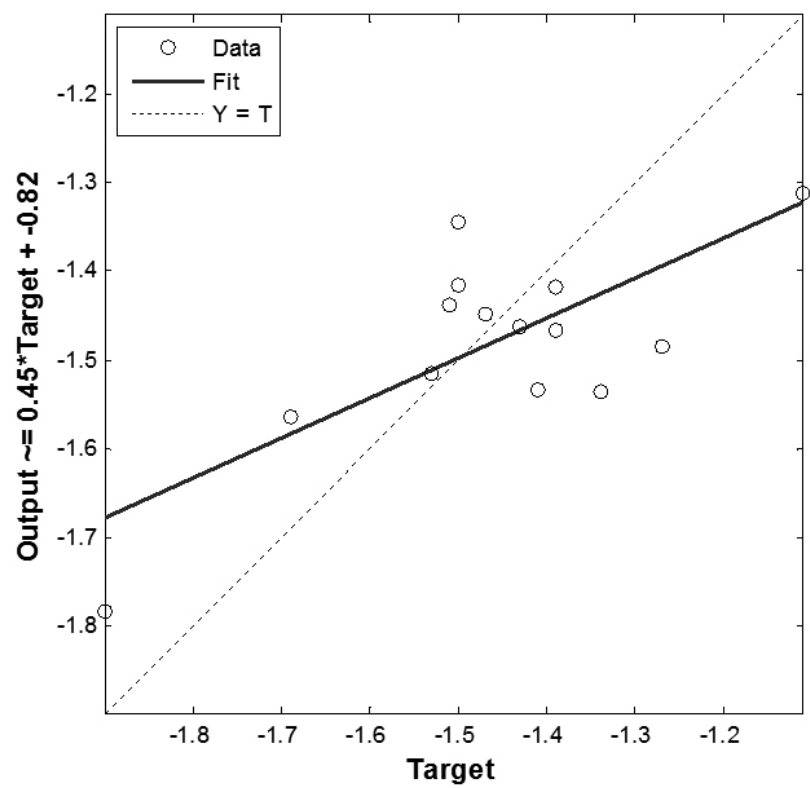

Fig. 3. Plot of output versus target data using ICA method.

RESULTS AND DISCUSSION

In this work, QSAR between oral bioavailabilities of some drugs and their molecular structural descriptors were investigated by using linear and nonlinear techniques. After calculations of descriptors, the different methods were performed on the remaining descriptors to select the most important of them.

Statistical parameters of the different QSAR models are shown in Table 2. It can be seen that the RMSE values for the ANN and ICA method are better than those for the other methods. The descriptors selected using the methods described above were used to construct linear and nonlinear models using GA, ICA, ANN, MLR, PLS and PCR.

The efficiency of the QSAR model for predicting $\log \left(\mathrm{IC}_{50}\right)$ was estimated using the internal cross-validation method which resulted a prediction for log (1/IC50) using MLR, PLS and PCR (Table 1). Considering experimental error, the overall prediction for $\log (1 / \mathrm{IC} 50)$ was satisfactory.

Table1 shows the linear variable selection methodsused to select the most significant descriptors. The most significant descriptors selected are G3u, HATS2e, F02(C-N), GGI10, RDF040m, Mor22p, Mor05p, TIC4, H4e, H-052, G2m and G1e (Table 4).

Atomic masses, symmetry directional WHIM index, electronegativities, frequency of $\mathrm{C}-\mathrm{N}$ at topological distance, radial distribution function, atomic van der Waals volumes, atomic polarizabilities and symmetry were important descriptors in this study.

Atom polarizabilities are linearly correlated with their hardness. The atomic properties considered are partial charges, electron densities and polarizabilities, calculated by computational chemistrymethods; moreover, bond properties have been proposed as the difference between the property values of the atoms forming the bond. $G G_{k}$ is The topological charge index .

The radial distribution function (RDF) descriptors are based on the distance distribution in the molecule. The radial distribution function of an ensemble of $n$ atoms can be interpreted as the probability distribution of finding an atom in a spherical volume of radius $R$.

3D MoRSE descriptors (3D Molecule Representation of Structures based on Electron diffraction) are derived from Infrared spectra simulation using a generalized scattering function.

WHIM descriptors are based on the statistical indices calculated on the projections of atoms along principal axes. They are built in such a way as to capture relevant molecular 3D information regarding the molecular size, shape, symmetry and atom distribution with respect to invariant reference frames. The algorithm consists of performing a Principal Components Analysis on the centered Cartesian coordinates of a molecule by using a weighted covariance matrix obtained from different weighing schemes for the atoms.

HATS descriptors are computed on a Hydrogen-filled molecule. We construct the Molecular Influence matrix $H$ as follows. Let $M$ be the geometric distance matrix having $n$ rows and 3 columns, where we have one row for each of the $n$ atoms present in the molecule and one column for each of the $\mathrm{x}-, \mathrm{y}-, \mathrm{z}-$ coordinates of the atoms in the molecule. The atomic coordinates are assumed to be calculated with respect to the geometric center of the molecule.

Bond character is closely related to the capacity of bonded atoms to exchange electrons, and such capacity is commonly well represented by the electronegativity $\mathrm{x}$ of the bonded atoms.

The molecular weight can be viewed as a simple linear atom contribution model, where the group contributions are atomic masses. In the first case, large training sets are used to obtain reliable estimates of the group contributions. Usually a battery of group contributions (a field of scalar parameters) is defined taking into account several structural characteristics of the molecules, also sometimes adding extra terms (correction factors) referring to special substructures.

$\mathrm{R}^{2}=0.73$ for the stepwise-MLR model. The result of the ICA was Output $=0.28$ Target +1.1 with a training $\mathrm{R}$ equal to 0.74 (Figure 3 ).

For MLR, we found that: Out $=0.841-4.807 \mathrm{G} 3 \mathrm{u}-8.655 \mathrm{Gle}$

For MLR-ICA, we had: Out: $=0.386+0.39 \mathrm{Le} 3-0.496$ Mor $32 \mathrm{~m}$

For MLR-GA, we found that: Out $=-1.115-10.104 \mathrm{G} 1 \mathrm{e}+7.301 \mathrm{G} 2 \mathrm{v}$

As can be seen in this table, there is correlation between selected molecular descriptors [Table5]. We have correlation:

HATS2e with Mor05p

Mor32m with Mor05p

G1e with Mor $32 \mathrm{~m}$

F02(C-N) with H052

RDF040m with TIC4

F02(C-N) with Mor05p

In studies with different methods and different goals, compounds $1,4,11$, 15 and 17 among 21 studied compounds have the lowest deviation and are suggested as the best compounds to make anti-TB drugs.

Table 1.Experimental and predicted values of $\log \left(1 / \mathrm{IC}_{50}\right)$ using Jack Knife, PLS PCR model.

\begin{tabular}{|c|c|c|c|c|}
\hline Sample & $\begin{array}{c}\text { Observed } \\
\log \left(1 / \mathrm{IC}_{50}\right)\end{array}$ & $\begin{array}{c}\text { Calculated } \\
(\text { Jack-knife })\end{array}$ & $\begin{array}{c}\text { Calculated } \\
\text { GA-PCR }\end{array}$ & $\begin{array}{c}\text { Calculated } \\
\text { GA-PLS }\end{array}$ \\
\hline 1 & -1.4100 & -1.3947 & -1.592 & -1.570 \\
\hline 2 & -1.1100 & -1.5221 & -1.349 & -1.316 \\
\hline 3 & -1.4700 & -1.4271 & -1.412 & -1.419 \\
\hline 4 & -1.5000 & -1.4162 & -1.392 & -1.347 \\
\hline 5 & -1.5300 & -1.4322 & -1.512 & -1.507 \\
\hline 6 & -1.5000 & -1.3818 & -1.372 & -1.353 \\
\hline 7 & -1.5100 & -1.4358 & -1.312 & -1.333 \\
\hline 8 & -1.3900 & -1.4375 & -1.276 & -1.277 \\
\hline 9 & -1.2700 & -1.4458 & -1.473 & -1.497 \\
\hline 10 & -1.3900 & -1.4400 & -1.458 & -1.462 \\
\hline 11 & -1.4300 & -1.4475 & -1.540 & -1.540 \\
\hline 12 & -1.3400 & -1.4657 & -1.541 & -1.550 \\
\hline 13 & -1.9000 & -1.5433 & -1.680 & -1.710 \\
\hline 14 & -1.6900 & -1.3898 & -1.512 & -1.524 \\
\hline 15 & -1.4400 & -1.4657 & -1.480 & -1.484 \\
\hline 16 & -1.9000 & -1.5183 & -1.569 & -1.572 \\
\hline 17 & -1.6500 & -1.4101 & -1.615 & -1.637 \\
\hline 18 & -1.7000 & -1.4023 & -1.599 & -1.610 \\
\hline 19 & -1.3200 & -1.4303 & -1.527 & -1.479 \\
\hline 20 & -1.3400 & -1.4413 & -1.403 & -1.416 \\
\hline 21 & -1.3200 & -1.4308 & -1.495 & -1.505 \\
\hline & & & & \\
\hline
\end{tabular}


J. Chil. Chem. Soc., 63, No 4 (2018)

Table 2. The statistical parameters of different constructed QSAR models.

\begin{tabular}{|c|c|c|c|c|c|c|c|c|}
\hline Methods & RMSE $_{1}$ & $\mathrm{RMSE}_{2}$ & $\mathrm{R}^{2}$ & $\mathrm{P}$ value & Fratio & $\begin{array}{l}\text { Standard error of } \\
\text { predicted value }\end{array}$ & $\mathrm{Q}^{2}$ & $\mathrm{R}^{2}$ pre \\
\hline Jack-knife & 0.1267 & 0.1395 & - & - & - & - & 0.94 & \\
\hline ICA & - & 0.1728 & - & - & - & - & & \\
\hline LASSO & - & - & 0.93 & - & - & 0.027 & & \\
\hline GA-MLR & - & - & 0.73 & - & - & - & & \\
\hline GA-PLS & 0.0018 & 0.0235 & 0.81 & - & - & - & 0.52 & 0.68 \\
\hline GA & 0.1350 & 0.1748 & - & - & - & - & & \\
\hline $\mathrm{RS}$ & - & - & - & 0.04 & 1.7 & - & & \\
\hline
\end{tabular}

Table 3. Descriptors values for GA.

\begin{tabular}{|c|c|c|c|c|c|c|c|}
\hline Molecule & TIC4 & Mor22p & $\mathrm{H} 4 \mathrm{e}$ & H-052 & $\mathrm{G} 2 \mathrm{~m}$ & G1e & $\mathrm{F} 02[\mathrm{C}-\mathrm{N}]$ \\
\hline 1 & 294.896 & 0.435 & 2.800 & 2 & 0.165 & 0.151 & 5 \\
\hline 2 & 311.908 & 0.229 & 2.753 & 2 & 0.186 & 0.154 & 4 \\
\hline 3 & 332.163 & -0.185 & 2.907 & 4 & 0.155 & 0.149 & 5 \\
\hline 4 & 291.415 & 0.662 & 2.671 & 1 & 0.156 & 0.142 & 4 \\
\hline 5 & 268.226 & 0.471 & 3.289 & 4 & 0.152 & 0.159 & 5 \\
\hline 6 & 360.725 & -0.009 & 3.118 & 2 & 0.152 & 0.139 & 4 \\
\hline 7 & 384.897 & 0.045 & 3.198 & 5 & 0.147 & 0.144 & 5 \\
\hline 8 & 363.970 & -0.101 & 2.903 & 4 & 0.152 & 0.146 & 5 \\
\hline 9 & 358.928 & 0.106 & 3.247 & 4 & 0.154 & 0.154 & 5 \\
\hline 10 & 312.226 & 0.226 & 3.114 & 4 & 0.181 & 0.163 & 5 \\
\hline 11 & 272.904 & -0.057 & 2.411 & 5 & 0.190 & 0.153 & 5 \\
\hline 12 & 287.394 & 0.141 & 2.920 & 5 & 0.160 & 0.160 & 5 \\
\hline 13 & 252.253 & 0.557 & 2.613 & 6 & 0.154 & 0.169 & 6 \\
\hline 14 & 298.149 & 0.143 & 2.506 & 5 & 0.178 & 0.167 & 5 \\
\hline 15 & 284.265 & -0.028 & 2.819 & 5 & 0.177 & 0.159 & 5 \\
\hline 16 & 270.457 & 0.434 & 2.387 & 4 & 0.147 & 0.163 & 5 \\
\hline 17 & 281.455 & 0.291 & 2.444 & 6 & 0.174 & 0.159 & 6 \\
\hline 18 & 303.510 & 0.218 & 2.494 & 4 & 0.148 & 0.166 & 6 \\
\hline 19 & 311.735 & -0.070 & 2.181 & 0 & 0.150 & 0.143 & 5 \\
\hline 20 & 302.654 & 0.288 & 3.316 & 5 & 0.167 & 0.155 & 5 \\
\hline 21 & 300.743 & -0.020 & 2.383 & 5 & 0.177 & 0.146 & 5 \\
\hline
\end{tabular}

Table 4. The mean of selected Descriptors.

\begin{tabular}{|c|c|c|c|}
\hline Descriptor Symbol & Descriptor group & Meaning & Method \\
\hline MATS6m & 2D autocorrelations & Morgan autocorrelation-lag6/weighted by atomic masses & LASSO \\
\hline $\mathrm{G} 3 \mathrm{u}$ & WHIM & 3 st component symmetry directional WHIM index & LASSO \\
\hline HATS2e & GETAWAY & $\begin{array}{c}\text { Leverage-weighted autocorrelation of lag } 2 / \text { weighted by atomic Sanderson } \\
\text { electronegativities }\end{array}$ & LASSO \\
\hline $\mathrm{F} 02(\mathrm{C}-\mathrm{N})$ & 2D frequency finger prints & frequency of $\mathrm{C}-\mathrm{N}$ at topological distance 02 & LASSO \\
\hline GGI10 & Topological & Topological charge index of order 10 & ICA \\
\hline RDF040m & RDF & Radial Distribution function -4/Weighted by atomic masses & ICA \\
\hline Mor22p & 3D-MoRSE & 3D-MoRSE-signal22/ Weighted by atomic masses & ICA,GA-ANN \\
\hline Mor05p & 3D-MoRSE & 3D-MoRSE-signal05/ Weighted by atomic polarisabilities & ICA \\
\hline TIC4 & Information indices(2D) & Total information content index (neighborhood symmetry of 4-order ) & GA-ANN \\
\hline $\mathrm{H} 4 \mathrm{e}$ & GETAWAY & H autocorrelation of lag 4 / weighted by atomic Sanderson electronegativities & GA-ANN \\
\hline H-052 & Atom-centered fragments (1D) & $\mathrm{H}$ attached to $\mathrm{C} 0(\mathrm{sp} 3)$ with $1 \mathrm{X}$ attached to next $\mathrm{C}$ & GA-ANN \\
\hline G1e & WHIM & $\begin{array}{l}\text { 1st component symmetry directional WHIM index/weighted by atomic } \\
\text { Sanderson electronegativities }\end{array}$ & GA-MLR \\
\hline $\mathrm{G} 2 \mathrm{v}$ & WHIM & $\begin{array}{l}\text { 2st component symmetry directional WHIM index/weighted by atomic van der } \\
\text { waals valumes }\end{array}$ & GA-MLR \\
\hline G3u & WHIM & 3st component symmetry directional WHIM index/Unweighted & MLR \\
\hline Le3 & WHIM & $\begin{array}{l}\text { 3rd component symmetry size directional WHIM index/ weighted by atomic } \\
\text { Sanderson electronegativities }\end{array}$ & MLR-ICA \\
\hline Mor32m & 3D-MoRSE & 3D-MoRSE signal 32/ weighted by atomic masses & MLR-ICA \\
\hline
\end{tabular}


Table 5. Correlation matrix between selected descriptors.

\begin{tabular}{|c|c|c|c|c|c|c|c|c|c|c|c|c|c|c|c|c|}
\hline & $\begin{array}{c}\text { TIC } \\
4 \\
\end{array}$ & $\begin{array}{c}\text { MATS } \\
6 \mathrm{~m}\end{array}$ & $\begin{array}{l}\text { GG } \\
\mathrm{I} 10\end{array}$ & $\begin{array}{l}\text { RDF } \\
040 \mathrm{~m}\end{array}$ & $\begin{array}{l}\text { Mor } \\
32 \mathrm{~m}\end{array}$ & $\begin{array}{c}\text { Mor } \\
05 p\end{array}$ & $\begin{array}{l}\text { Mor } \\
22 p\end{array}$ & $\begin{array}{c}\mathrm{G} \\
3 \mathrm{u}\end{array}$ & $\begin{array}{c}\mathrm{G} \\
2 \mathrm{~m}\end{array}$ & $\begin{array}{l}G \\
2 v\end{array}$ & $\begin{array}{c}\text { Le } \\
3\end{array}$ & G1e & $\begin{array}{l}\mathrm{H} \\
4 \mathrm{e}\end{array}$ & $\begin{array}{c}\text { HATS } \\
2 \mathrm{e}\end{array}$ & $\begin{array}{c}\text { H0 } \\
52 \\
\end{array}$ & $\begin{array}{c}\mathrm{F} 02 \\
\left(\mathrm{C} \_\mathrm{N}\right)\end{array}$ \\
\hline TIC4 & 1 & & & & & & & & & & & & & & & \\
\hline MATS6m & 0.19 & 1 & & & & & & & & & & & & & & \\
\hline GGI10 & 0.33 & 0.07 & 1 & & & & & & & & & & & & & \\
\hline RDF040m & 0.65 & -0.01 & 0.30 & 1 & & & & & & & & & & & & \\
\hline Mor32m & -0.47 & -0.9 & -0.2 & -0.37 & 1 & & & & & & & & & & & \\
\hline Mor05p & -0.65 & -0.22 & -0.37 & -0.65 & 0.56 & 1 & & & & & & & & & & \\
\hline Mor $22 p$ & -0.53 & -0.35 & -0.26 & -0.11 & 0.25 & 0.09 & 1 & & & & & & & & & \\
\hline G3u & -0.25 & -0.19 & -0.49 & -0.33 & 0.20 & 0.21 & 0.45 & 1 & & & & & & & & \\
\hline $\mathrm{G} 2 \mathrm{~m}$ & -0.32 & 0.18 & 0.10 & -0.08 & 0.09 & 0.16 & -0.11 & -0.31 & 1 & & & & & & & \\
\hline $\mathrm{G} 2 \mathrm{v}$ & 0.01 & 0.05 & 0.25 & 0.14 & -0.18 & -0.05 & -0.4 & -0.37 & 0.63 & 1 & & & & & & \\
\hline Le3 & 0.21 & 0.08 & 0.06 & 0.06 & -0.67 & -0.13 & -0.29 & -0.04 & 0.03 & 0.1 & 1 & & & & & \\
\hline G1e & -0.52 & -0.25 & -0.07 & -0.51 & 0.67 & 0.59 & 0.36 & 0.25 & 0.24 & 0.07 & -0.48 & 1 & & & & \\
\hline $\mathrm{H} 4 \mathrm{e}$ & 0.35 & -0.08 & 0.20 & 0.32 & 0.06 & -0.37 & 0.06 & 0.1 & -0.06 & 0.06 & 0.23 & 0.04 & 1 & & & \\
\hline HATS2e & -0.83 & -0.42 & -0.37 & -0.57 & 0.25 & 0.69 & 0.50 & 0.28 & 0.24 & -0.18 & -0.11 & 0.5 & -0.3 & 1 & & \\
\hline H052 & -0.38 & 0.06 & -0.06 & -0.29 & 0.15 & 0.49 & 0.03 & -0.10 & 0.29 & 0.27 & -0.09 & 0.44 & -0.17 & 0.37 & 1 & \\
\hline F02 (C_N) & -0.37 & -0.11 & -0.21 & -0.27 & 0.61 & 0.63 & 0.12 & 0.13 & -0.02 & 0.12 & -0.43 & 0.61 & -0.16 & 0.19 & 0.61 & 1 \\
\hline
\end{tabular}

\section{CONCLUSION}

In the present study, MLR, PLS ,GA, ANN, ICA and ANN were used as linear and nonlinear models to their calculated molecular descriptors. The calculated statistical parameters of these models revealed that ANN was better than others which means that there are some linear and nonlinear relations between selected molecular descriptors and their structures. ANN and ICA were successfully used to develop a QSAR model for ethylene diamine derivatives that provided the best results in comparison with other methods. This attempt to correlate $\log \left(1 / \mathrm{IC}_{50}\right)$ with theoretically calculated molecular descriptors led to a relatively successful QSAR model that relates these derivatives.

CONFILICT OF INTEREST: The authors have no conflict of interest.

\section{REFERENCES}

[1] F. J. DuMelle, P. C. Hopewell, TB Notes News let. 1, 23-27. (2000)

[2] J. B . Jr.Bass, L. S . Farer, P. C. Hopewell , Am. J. Respir. Crit Care Med .149, 1359-1374. (1994)

[3] C. R. Jr.Horsburgh , S. Feldman, R. Ridzon, Clin. Infect Dis . 31, 633639. (2000)

[4] P. A. Gross, T. L. Barrett , E. P. Dellinger, P. J. Krause , W. J. Martone, J. E. Jr. McGowan, R. L. Sweet, R. P. Wenzel, Clin. Infect Dis . 18, 421. (1994)

[5] R. Hosseini, H. Salehipoor, Int. J. Struct. Stab. Dy . 12, No. 3, 1250019 . (2012)

[6] E. Atashpaz-Gargari , C. Lucas. Imperialist competitive algorithm: an algorithm for optimization inspired by imperialistic competition. Evolutionary Computation, CEC 2007. IEEE Congress on , 2007; pp. 4661-4667.

[7] E. Atashpaz-Gargari , C. Lucas. Designing an optimal PID controller using Colonial Competitive Algorithm. First Iranian Joint Congress on Intelligent and Fuzzy Systems, 2007.

[8] C. Sarbu, C. Onisor, M. Poša, Talanta .75, 651-657. (2008)

[9] M. P. Freitas, J. A. Martins, Talanta. 67, 182-186. (2005 )

[10] K. Valko, J. Chromatog. A. 1037, 299-310. (2004)

[11] K. Tang, T. Li, Anal. Chem. Acta. 476 , 75-92. (2003)

[12] H. Gonzalez-Diaz, I. Bonet, C. E. Teran, Eur. J. Med.Chem. 42, 580585. (2007)

[13] S. Vilar, L. Santana, E. Uriarte, J. Med. Chem. 49, 1118-1124. (2006)

[14] C. Tang, P. A. Almeida Fishwick Times series forecasting using neural networks vs. Box-Jenkins methodologySimulations, Simulations Councils, 1991; pp. 303-310.
[15] Marina. Protopopova, Colleen. Hanrahan, Boris Nikonenko, J. Antimicrob. Chemother . 56, 968-974. (2005)

[16] R. Todeschini, V. Consonni, Handbook of Molecular Descriptors; WILEY-VCH, Verlag GmbH. Vol. 11, 2000; p 516. 